\title{
EL ENTE Y SU NATURALEZA
}

ADOLFo GaRcía DE LA SiENRA*

CENTRO DE INVESTIGACIÓN Y DOCENCIA ECONÓMICAS

\section{Introducción}

La experiencia preteórica reconoce intuitivamente la gran diversidad de los seres que nos rodean. Dentro de la tradición filosófica occidental, desde la antigua Grecia, ha habido un persistente intento por dar cuenta de esta diversidad mediante los conceptos de esencia y forma sustancial. La finalidad de este artículo es introducir una explicación diferente, una explicación que rompe con el esquema aristotélico-tomista, al mismo tiempo que busca hacer justicia al hecho de que tal diversidad no es producto de la actividad arbitraria del hombre.

\section{El orden modal}

Si bien los filósofos desde hace tiempo han admitido que no es factible reducir todas las leyes del cosmos a un solo nivel o tipo de ley, se debe a Herman Dooyeweerd el haber enfatizado el orden modal del cosmos y el carácter interdependiente de todos los niveles o modalidades. Mario Bunge, por ejemplo, admite que

una hipótesis ontológica involucrada en la ciencia moderna y alentada por ella es que la realidad, tal y como nos es conocida hoy, no es un sólido bloque homogéneo sino que está dividida en varios niveles, o sectores, cada uno caracterizado por un conjunto de propiedades y leyes que le son propias. Los principales niveles reconocidos en la actualidad parecen ser el físico, el biológico, el psicológico, y los socioculturales. Cada uno de éstos puede a su vez ser dividido en subniveles. Por ejemplo, los principales subniveles del nivel físico son los niveles físico propiamente dicho y químico, y los principales subniveles del nivel sociocultural son el económico, el social, y los subniveles culturales. Divisiones más finas pueden ser introducidas y ninguna es rígida ni está delineada claramente. ${ }^{1}$

* Este trabajo fue elaborado con el apoyo del proyecto L6́gica y ontología, IN-601393, de la DGAPA-UNAM.

1 Bunge (1967), p. 293. La traducción es mía. 
Estos "niveles" o "sectores" han sido llamados modalidades o aspectos de la experiencia por Dooyeweerd y en buena medida coinciden puntualmente con los que Bunge reconoce. Aparte de los mencionados por Bunge, Dooyeweerd incluye "más abajo" los niveles o modalidades aritmético, espacial y cinemático; "más arriba" incluye la modalidad lógica y sobre ésta, en el "nivel cultural", incluye las modalidades histórica o cultural propiamente dicha, la lingual, la social, la económica, la estética, la diquética, la ética y la fídica (véase la tabla). ${ }^{2}$

\section{MODALIDADES O ASPECTOS DE LA EXPERIENCIA}

\section{Aspectos}

1 Aritmético (numérico)

2 espacial

3 cinemático

4 físico

5 biótico

6 sensitivo (síquico)

7 lógico

8 histórico

9 lingual

10 social

11 económico

12 estético

13 diquético

14 ético

15 fídico
Significados nucleares

cantidad discreta (número)

extensión

movimiento (locomoción)

energía

vitalidad (vida)

sentimiento, percepción sensorial

distinción analítica

poder formativo

significado simbólico

relaciones sociales

frugalidad en el manejo

de bienes escasos

armonía

retribución (recompensa)

amor en las relaciones temporales

fe, firme seguridad

Basado en Kalsbeek (1975), p. 100.

La filosofía de la idea cosmonómica sostiene que las modalidades son precisamente las quince enlistadas y que éstas están dadas en un orden lineal, que llamaremos cadena modal. Si $M$ es un elemento de esta cadena, llamaremos modalidad sustrato a cualquier modalidad que anteceda a $M$ en dicho orden lineal (si es que hay alguna); asimismo, llamaremos modalidad superestrato a cualquier modalidad que suceda a $M$ en el mismo orden (si es que hay alguna). La primera y última modalidades de la cadena serán llamadas modalidades terminales. Así, la primera modalidad terminal es la aritmética, mientras que la última es la pística. A la dirección del orden de

2 Dooyeweerd (1985), v. 1, p. 1. Véanse los detalles en el vol. 2. 
la primera a la última se le llama dirección trascendental, mientras que su inversa se conoce como dirección fundacional.

Es posible discutir si éstas son todas o algunas de las componentes del orden modal del ser, pero parece claro que difícilmente se puede negar dicho orden, por más que los filósofos hayan intentado eliminar algunos de esos aspectos en favor de otros. Las diferencias sustanciales aparecen cuando se trata de establecer el status ontológico de dichas modalidades, es decir, las relaciones de dependencia existencial que tienen lugar entre ellas. Hay tres posiciones principales en este respecto, las que llamaré "reduccionismo fuerte", "reduccionismo débil" y "antirreduccionismo cosmonómico".

El reduccionismo fuerte parte de la admisión prima facie del orden modal pero se propone demostrar que la supuesta soberanía de algunas modalidades es aparente, en el sentido de que se trata de leyes o propiedades analizables o eliminables en términos de modalidades de estratos inferiores o superiores. El fisicalismo en filosofía de la mente es un ejemplo de reduccionismo en sentido fuerte, pues pretende eliminar una modalidad determinada en aras de modalidades sustrato al tratar de reducir las propiedades síquicas a propiedades puramente físicas o bióticas. Otro ejemplo de reduccionismo fuerte, en sentido inverso, es el logicismo, el cual se propone reducir la primera modalidad terminal a una de sus modalidades superestrato, la modalidad lógica.

El reduccionismo débil no pretende eliminar las propiedades de una modalidad en aras de las de otra(s), sino tan sólo defender que algunas son básicas con respecto a las demás, en el sentido de que las modalidades básicas pueden existir sin las otras, pero las otras no pueden existir sin las básicas. Ejemplo de esta forma de reduccionismo es una segunda presuposición de muchos científicos y filósofos que muchas veces acompaña a la primera presuposición. Como dice Bunge:

Una segunda presuposición, relacionada, es que los niveles más altos están enraizados en los más bajos, tanto histórica como contemporáneamente: esto es, los niveles más altos no son autónomos sino que dependen para su existencia de la subsistencia de los niveles más bajos, y han emergido en el curso del tiempo de los más bajos en un número de procesos evolutivos. Este enraizamiento de lo más alto en lo más bajo es la base objetiva de la posibilidad de explicar parcialmente lo más alto en términos de lo más bajo o viceversa ${ }^{3}$

Esta posición es la del mismo Bunge y ha sido asumida y llamada 'materialismo irreductivo' por Ingvar Johansson (1989). El antirreduccionismo cosmonómico rechaza ambas posiciones, pues considera que las modalidades son existencialmente interdependientes de modo que ninguna es ontologicamente más básica que las demás. El antirreduccionismo cosmonómico ve

3 Ibid. La traducción es mía. 
la cadena modal como un todo indestructible que es a la vez uno de los aspectos del cosmos. Este aspecto se conoce como el lado ley del cosmos. El otro aspecto se denomina el lado sujeto del cosmos. Estos aspectos están indisolublemente unidos, de modo que es imposible que exista el uno sin el otro, aunque esto no quiere decir que, en cualquier momento del devenir del universo propiedades de todas las modalidades están siendo ejemplificadas en acto por algún ente individual.

En efecto, la filosofía de la idea cosmonómica coincide con el materialismo irreductivo en que individuos de diferentes clases fueron apareciendo a lo largo del proceso de devenir, de manera que las propiedades de modalidades superiores - de la lógica en adelante- no fueron ejemplificadas en acto antes de la aparición del hombre. Pero esto no equivale a decir que las modalidades sustrato tengan un status ontológico privilegiado, o que las modalidades superestrato hayan emergido en el curso del tiempo de las más bajas en un "número de procesos evolutivos". Tampoco se sigue que este supuesto enraizamiento de las modalidades más altas en las más bajas sea "la base objetiva de la posibilidad de explicar parcialmente lo más alto en términos de lo más bajo o viceversa".

Roy Clouser (1991) ha argumentado la inviabilidad de las estrategias reduccionistas, que buscan privilegiar unas modalidades en detrimento de otras. Ha mostrado que las estrategias reduccionistas no sólo son teoréticamente injustificables, sino sobre todo autorrealizativamente incoherentes (self-performatively incoherent), pues

abstraer cualquier aspecto de la experiencia y proclamarlo autoexistente es hacer una aseveración autorrealizativamente incoherente. ${ }^{4}$

Un procedimiento que permite visualizar la inconveniencia del reduccionismo consiste en darse cuenta de que ninguna modalidad es inteligible sin las otras. Pretender, por ejemplo, que la modalidad física puede existir sin sus superestratos, equivale a postular la posibilidad real de una Ding an sich física; esto es, la posibilidad de un cuerpo físico enteramente carente de propiedades sensoriales (imperceptible), lógicas (ininteligible), históricas (incapaz de ser usado en la producción de artefactos), etcétera. Pero de hecho - sostengo- las cosas físicas no son así, sino que tienen en potencia (o en acto) propiedades de las modalidades superiores, razón por la cual pueden funcionar, y de hecho funcionan, en la coherencia de la experiencia humana.

Otro argumento que puede ser esgrimido en contra del materialismo reductivo es el siguiente. Los materialistas reductivos pretenden que las leyes de las modalidades superiores surgen cuando aparecen, mediante procesos 195.

4 Véanse los detalles del argumento en el texto citado, particularmente en las pp. 191- 
evolutivos, los entes que las ejemplifican. Esto implica que un proceso evolutivo que genera un ente que obedece leyes de una modalidad no existente hasta el momento no está sujeto a dichas leyes. Luego entonces, un proceso evolutivo que genera un ser vivo por primera vez es (digamos) un proceso puramente físico, pues ex hypothesi no hay leyes bióticas; pero a la vez no es puramente físico, pues posee ciertamente por lo menos la propiedad biótica (nada desdeñable) de ser capaz de generar un ser vivo. Muchos opinan que pensar así es el único camino de la ciencia, pero a mí más bien me parece pura magia.

Me parece más sensato decir, como Dooyeweerd, que el devenir del cosmos funciona simultáneamente en todas las modalidades, lo cual presupone que el orden modal está dado con el cosmos desde un principio, precisamente como su lado ley. Desde su misma creación, el proceso de devenir del cosmos está regido por leyes que establecen el orden causal dentro del que van surgiendo ejemplares que pertenecen a clases naturales no anteriormente ejemplificadas. Si se me permite una metáfora: en un feto (humano) no se puede apreciar su sujeción a leyes psíquicas, lógicas o linguales, no por ello deja de estar sujeto a ellas. Sólo en relación con las propiedades lógicas, sociales y culturales aunque se entiende el significado del feto (a saber, como un ser cuyo desarrollo naturalmente debe culminar en un ser humano). Así, sólo por virtud de dichas propiedades se entiende el devenir del cosmos y se explica que hayan surgido las diversas formas minerales, bióticas, animales y sociales. Esta tesis debe ser contrastada con el evolucionismo. Lo que éste sostiene es que el surgimiento de "propiedades supervinientes" bióticas no está regido por ley alguna que involucre estas propiedades. Esta tesis difícilmente inteligible - ha sido refinada usando ideas informáticas. Así, algunos evolucionistas se imaginan que las diversas formas bióticas son el despliegue de un programa semejante a un software tan sofisticado que es capaz de crear nuevos programas inteligentes, lo cual desde luego plantea la pregunta relativa al origen de dicho programa. Lógicamente, sólo hay dos respuestas posibles y ambas son igualmente injustificables: una respuesta es que el mismo es increado, la otra es que es creado. La primera respuesta satisface el motivo religioso (ateo) del evolucionismo; la segunda se aproxima más al motivo religioso (teísta) de la filosofía de la idea cosmonómica. Metafóricamente hablando, un programa como ése sería el lado ley del cosmos, mientras que las formas individuales a las que va dando lugar constituirian el lado sujeto. Pero sea creado o increado, seguramente un programa tal contendría ya la coherencia de las leyes y propiedades pertenecientes a las diferentes modalidades, y eso era todo el punto.

Metáforas aparte, no es posible entender el surgimiento de las diferentes formas bioticas y culturales sin un marco nómico que rija dicho desarrollo. El orden causal no puede ser concebido al margen de este marco. Pero antes de pasar al tema de la causalidad cabe decir que, según la filosofía de 
la idea cosmonómica, la base "objetiva" que permite explicar parcialmente las modalidades superiores en términos de las inferiores, o viceversa, no es que las segundas surjan de las primeras, sino precisamente la coherencia de las modalidades entre sí. Esta coherencia hace posible, asimismo, que propiedades o leyes pertenecientes a una modalidad sean representadas mediante propiedades o leyes pertenecientes a otra; esto hace posible, en particular, el modelamiento matemático de estructuras que en sí mismas no son ni numéricas ni espaciales. Estos modelamientos o representaciones no son sino analogias o expresiones del significado nuclear de una modalidad en el de otra. Por ejemplo, los positivistas críticos alemanes hablaban de economía del pensamiento. Hay una modalidad de leyes y propiedades propiamente económica, otra modalidad de leyes y propiedades propiamente lógicas, y cada una de estas modalidades tiene su propio significado nuclear: la frugalidad en el manejo de bienes escasos y la distinción analítica, respectivamente. ¿Qué tiene que ver una con la otra? Como lo dice Kalsbeek:

La frugalidad se origina en el aspecto económico; aquí es original. En los otros aspectos ocurre como un momento de significado, pero no en su sentido original. Cuando ocurre en los otros aspectos, la frugalidad siempre está caracterizada o cualificada por los núcleos de significado de los aspectos no económicos que así mantienen su soberanía de esfera modal. ${ }^{5}$

La analogía consiste en la modificación que del significado nuclear de la esfera económica hace el significado nuclear de la modalidad lógica. En otras palabras, si bien el significado nuclear de la modalidad lógica no es el manejo frugal de bienes escasos, la Navaja de Occam anticipa el significado nuclear de una esfera superestrato - la económica - al requerir el manejo eficiente de conceptos y premisas en el análisis y la argumentación. Hay aquí claramente, de hecho, una similitud de significado que expresa justamente la profunda coherencia de las modalidades entre sí. En términos generales, si la analogía consiste en el anuncio del significado nuclear de una modalidad superestrato en una modalidad determinada, entonces es llamada anticipación; si consiste en el anuncio del significado nuclear de una modalidad sustrato, se llama - si se me da licencia para introducir un neologismo técnico- retrocipación. Son estas analogías lo que permite explicar parcialmente propiedades pertenecientes a una modalidad en términos de otras. A este procedimiento lo llamo "representación", uno de cuyos ejemplos es lo que Krantz et al. (1971) llaman "medición fundamen-

5 Kalsbeek (1975), p. 105. La traducción es mía. 
tal"; o sea, el mapeo de propiedades de una modalidad en las modalidades aritmética y espacial. ${ }^{6}$

\section{La naturaleza de los entes}

La diversidad de los entes que constituyen el cosmos, el lado sujeto del mismo cosmos, está inextricablemente correlacionado con el orden modal. La tesis fundamental de la filosofía de la idea cosmonómica es que esta correlación consiste en que todos los entes individuales obedecen todas las leyes modales, o funcionan en todas las modalidades, si bien de dos maneras distintas. Unos entes funcionan activamente en unas modalidades, mientras que otros sólo pasivamente. La peculiar manera en que están organizadas las propiedades de un ente, esto es, la ley que lo define en su estructura y peculiar manera de operar, será llamada "ley tipo".

La ley tipo encierra la concepción cosmonómica de la naturaleza del ente en oposición a la aristotélico-tomista en particular. La principal diferencia radica en lo siguiente: (1) Se rechaza la explicación de la permanencia del ente en términos de una ousía o forma sustancial, pues ésta, al no ser concebida en el marco del orden modal, rompe la coherencia integral de los aspectos modales de la experiencia para dar lugar a la noción de sustancia como Ding an sich, es decir, como un ser independiente de una posible percepción o de la función lógica del pensamiento, como sujeto metafísico de propiedades pertenecientes a diferentes modalidades de la experiencia. ${ }^{7}$ (2) Se rechaza en consecuencia la noción de autosubsistencia del ente y se afirma que no hay nada increado en él, de modo que depende enteramente de Dios para subsistir. Pero no sólo eso: todo ente individual depende de su entorno para subsistir aunque sea genéricamente, como el hombre individual depende de ciertos tipos de cosas y procesos para vivir. (3) Ciertamente es necesario hablar del sustrato del cambio consistente en el venir a ser y dejar de existir de los entes, pero se rechaza la noción de materia prima como pura potencialidad. El sustrato último del cambio es él mismo un ente individual dotado con propiedades y que funciona en el orden modal de la experiencia. Como corolario, (4) se rechaza la noción de sustancia, pues el término sustancia viene usualmente cargado con una significación que implica las tesis que se acaban de rechazar, y no meramente -como a veces se afirma - la de sujeto de inherencia de propiedades o accidentes que no puede ser a su vez una propiedad. Esta última noción es desde

6 La figura, debida a José Antonio Vicuña (Doctorado en Finanzas Públicas, Universidad Veracruzana en Jalapa), muestra una representación espacial de la coherencia modal.

7 Considérese, como ejemplo, el alma como forma sustancial del hombre. Esta forma tiene propiedades como "racional", "sensible" o "vegetativa", y se supone que es el núcleo del hombre individual al estar unida a la materia. 


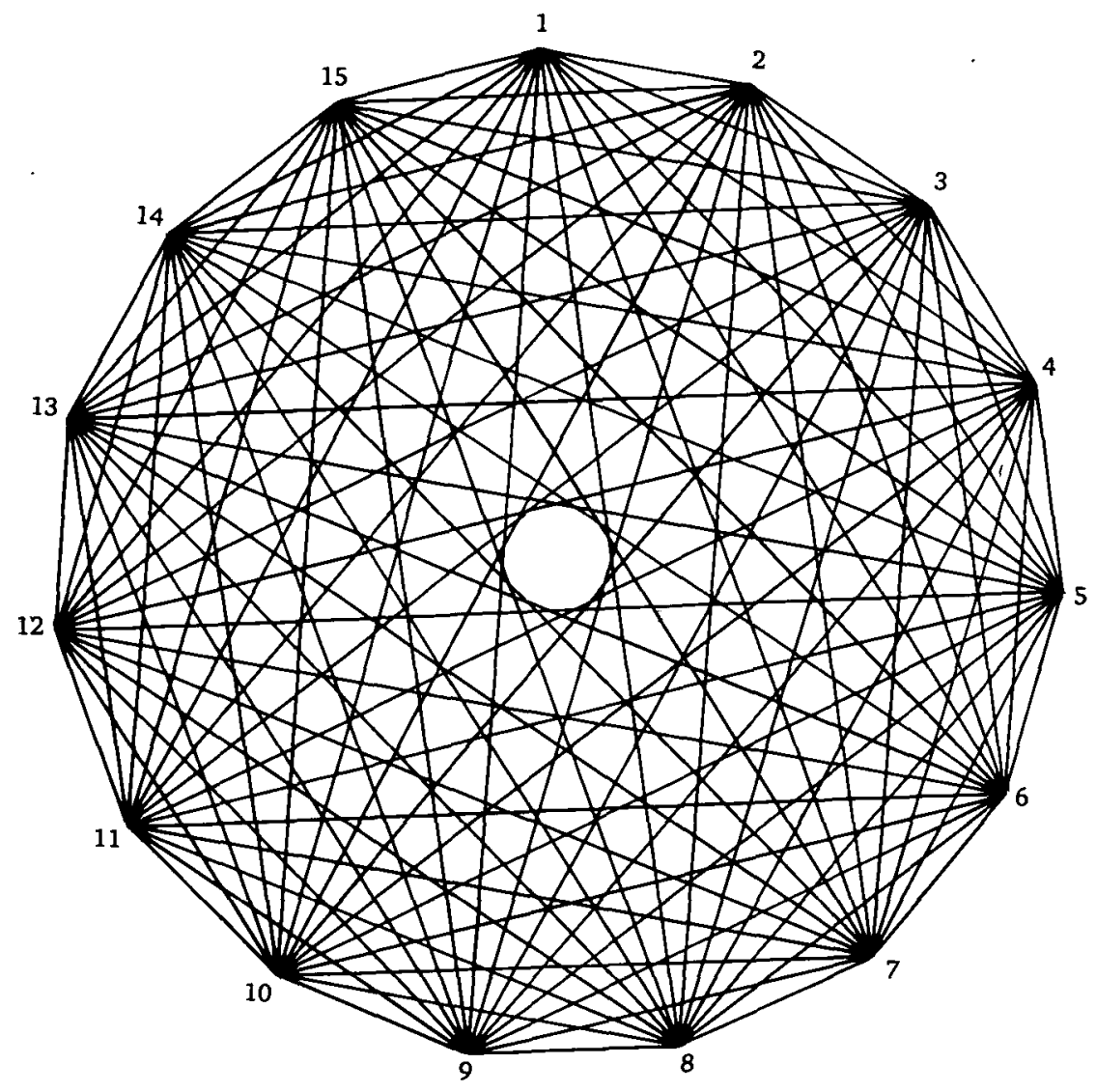

La coherencia modal. Cada modalidad está conectada con las demás. 
luego correcta e imprescindible, por lo que es necesario utilizar un término distinto para designar aquellos entes que no inhieren en otros.

Como la filosofía de la idea cosmonómica se reserva el término "sujeto" para designar a los entes en tanto que están sujetos a las leyes del cosmos, conviene designar a los entes que no son propiedades de otra manera. Lo que necesitamos es un término que signifique justamente 'no inherente en otro'. De hecho, un término con esta precisa significación fue utilizado por filósofos escolásticos como Francisco de Araújo, quien, según Beuchot, distinguió precisamente la razón que nos interesa entre cuatro nombres de la sustancia que significan "entitativamente" lo mismo. Este nombre es hipóstasis, "en cuanto le conviene el sustentar determinados accidentes, lo cual es un aspecto real". 8 Más precisamente, propongo definir hipóstasis como un ente individual al cual le conviene sustentar propiedades sin ser él mismo propiedad de ningún ente.

Para caracterizar la naturaleza o ley tipo de las hipóstasis, será de utilidad el concepto de dependencia existencial. Diremos que el ente $x$ es existencialmente dependiente del ente $y$ syss es cosmonómicamente imposible que $x$ exista si $y$ no existe. En términos de este concepto, podemos decir que $P$ es una propiedad intrínseca de una entidad $x$ syss $P$ es una propiedad de $x$ y $P$ y $x$ son mutuamente dependientes. Una propiedad intrínseca de un ente es aquélla la cual el ente no puede existir, y viceversa. Por ejemplo, este hombre no puede existir sin su capacidad de respiración, pero tampoco la capacidad de respirar de este hombre puede existir sin el hombre. Hago, en efecto, una distinción entre propiedades tipo y propiedades caso, de modo que los casos de propiedades que encontramos ejemplificadas en las cosas son ellas mismas individuos.

Si $x$ tiene una propiedad intrínseca $P$ perteneciente a una modalidad $M$, decimos que $x$ tiene una función sujeto en $M$, o que $x$ funciona activamente en $M$. Decimos que $x$ tiene una función objeto en $M$, o que $x$ funciona pasivamente en $M$ syss es cosmonómicamente posible que existan propiedades $P, Q_{1}, \ldots, Q_{n}$ pertenecientes a $M$, un ente $y, y$ una relación $R$ tal que $y$ funciona activamente en $M$ y $x$ tiene la propiedad $P$ syss $R x y$ y y tiene $Q_{1}, \ldots Q_{n}$ intrínsecamente. Decimos que $P$ es una propiedad potencial de $x$ mientras esta potencialidad no sea actualizada. Si la potencialidad es actualizada, decimos que la propiedad potencial $P$ ha sido activada por $Q_{1}, \ldots, Q_{n}$ y que $x$ tiene una función objeto activada en $M$. Para ilustrar estos conceptos, una roca, por ejemplo, tiene propiedades intrínsecas en las primeras cuatro modalidades, pero sólo propiedades potenciales en las otras, a menos que éstas sean activadas por su relación con plantas, animales o humanos. Así, la roca puede funcionar bióticamente como base para una planta en un barranco; 
psíquicamente como un resguardo seguro para un águila y su familia; o como un monumento histórico para los humanos.

Las propiedades intrínsecas de una entidad están siempre estructuradas de un peculiar modo nómico. Sean $P_{1}, \ldots, P_{n}$ las propiedades intrínsecas de una hipóstasis $x$. La ley tipo de $x$ es el arreglo nómico $L\left(P_{1}, \ldots, P_{n}\right)$ de estas propiedades. Si cualquier otra entidad $y$ tiene estas mismas propiedades bajo esta misma ley tipo, decimos que $x$ y $y$ son del mismo tipo. Por ende, las leyes tipo cruzan las modalidades determinando las combinaciones de propiedades que caracterizan a tipos de hipóstasis. Las propiedades intrínsecas de un ente están gobernadas por las leyes de una modalidad. Por ejemplo, las propiedades físicas o espaciales de un árbol están gobernadas por leyes bióticas: considérese que la forma del tronco está determinada por el peculiar modo en que el árbol organiza los tejidos conforme crece transformando los elementos nutritivos que obtiene del suelo. Por ello, entre las propiedades que constituyen la ley tipo de $x$, podemos distinguir un subconjunto de propiedades $Q_{1}, \ldots, Q_{m}(m<n)$, todas ellas pertenecientes a una y la misma modalidad, tal que su arreglo nómico $L\left(Q_{1}, \ldots, Q_{m}\right)$ determina la ley tipo de $x$, i.e. el peculiar modo en que están ordenadas todas las propiedades intrínsecas de $x$. Si $M$ es la modalidad a la cual pertenecen las propiedades $Q_{1}, \ldots, Q_{m}$, entonces decimos que $M$ es la función cualificadora de $x$.

Pero la ley estructural de una hipóstasis abarca mucho más que el ordenamiento de las propiedades del ente, ya que determina su estructura mereológica, esto es, los tipos de partes o subtodos esenciales que la constituyen, los tipos de hipóstasis con los que debe estar relacionada para poder subsistir (agua, aire, etcétera), así como los tipos de conexiones causales en las que puede entrar con las distintas clases de entes. Por ello, hay en su naturaleza una referencia explícita a las naturalezas de otras hipóstasis. Incidentalmente, esto constituye otro golpe mortal al concepto escolástico de causa formal o forma sustancial pues, como dice Gracia (1982),

por supuesto, las sustancias son de un modo $u$ otro dependientes de otras sustancias para su causación eficiente y, de acuerdo con los escolásticos, de Dios para su creación. Pero para su causación formal no dependen de nada extra, puesto que no hay referencia a otras sustancias o seres para su definición. ${ }^{9}$

Esta tesis es definitoria del concepto escolástico usual de sustancia y contrasta con la ley estructural que caracteriza el modo de ser de una hipóstasis, la cual hace referencia necesariamente a otras clases de hipóstasis por las razones expuestas. Al eliminarse el rígido concepto de forma sustancial en aras de uno que da cuenta no sólo del funcionamiento del ente en todas las

9 Gracia (1982), p. 268. 
modalidades, sino incluso de su estructura mereológica y de su estructura relacional en coherencia con otros tipos de hipóstasis, se ve todavía con mayor claridad por qué el concepto escolástico de sustancia es insostenible.

La estructura mereológica de un ente incluye no meramente su composición, sino también las clases de relaciones que rigen entre sus constituyentes. En términos muy generales, decimos que una hipóstasis $x$ de tipo $T$ (i.e. $T$ es la ley tipo de $x$ ) funciona en la organización interna de la hipóstasis $y$ syss es cosmonómicamente imposible que $y$ exista a menos que haya una relación $R$ tal que $R z y$ para alguna entidad $z$ de tipo $T$ o similar y, de hecho, $R x y$ y no $R w y$ para cualquier $w$ distinta de $x$. Decimos que $x$ es parte de $y$ syss (1) $x$ es existencialmente dependiente de $y$, (2) $x$ funciona en la organización interna de $y$, (3) $x$ tiene la misma función guía que $y$. El cerebro de un hombre, por ejemplo, es una parte del cuerpo de este hombre porque el primero no puede existir sin este cuerpo, funciona en la organización interna del cuerpo ( $R$ está ejemplificada como la relación ser el cerebro de), la función guía tanto del cuerpo como del cerebro es biótica. Decimos que una entidad $x$ tiene identidad individual syss no hay entidad $y$ tal que $x$ es parte de $y$. Se dice que una entidad $x$ es un subtodo de $y$ syss se cumple la condición (2) pero no la (1) o la (3).

Podemos decir ahora que una hipóstasis $x$ es una cosa natural syss $x$ funciona activamente en las primeras cuatro, cinco, o seis modalidades; esto es, si no hay un subtodo $y$ de $x$ tal que alguna propiedad intrínseca de $x$ es una propiedad activada de $y$. Una roca, por ejemplo, no tiene propiedades intrínsecas que sean el resultado de que cualquiera de sus subtodos esté relacionado con cualquier otra cosa: las propiedades de sus subtodos (incluyendo sus partes propiamente dichas), son todas intrínsecas a estos subtodos. Las hipóstasis que funcionan activamente en las primeras cuatro modalidades, pero no en las demás, constituyen el tipo radical ser inorgánico y el reino mineral. Las que funcionan activamente sólo en las primeras cinco son el tipo radical planta. Las que así funcionan sólo en las primeras seis son el tipo radical animal.

Un artefacto animal, en cambio, es una entidad tal que las propiedades no intrínsecas de algunos de sus subtodos naturales son activadas por la relación que el subtodo guarda con un animal. La guarida de un oso, por ejemplo, es un artefacto animal porque su subtodo (impropio), la cavidad en la roca misma, ha adquirido propiedades adicionales (olores, quizá ciertos acomodos en el suelo, qué sé yo), en relación con esta particular familia de osos. Como cualquier hipóstasis, la guarida tiene una función guía, pero - puesto que la guarida está de alguna manera fundada sobre una cosa natural, la cavidad en la roca- tendremos una mención especial a este fundamento y llamaremos función fundante de la guarida a la modalidad de su función guía. En términos generales, si $x$ es un subtodo natural de 
una artefacto animal $y$, decimos que aquella modalidad de $x$ en la que $x$ encuentra su función guia es una función fundante de $y$.

Los artefactos humanos son aún más complejos. Según Clouser (1991), hay un total de tres aspectos involucrados en la cualificación de un artefacto usual como un automóvil, a saber: el tipo de material natural, el tipo de proceso de producción, y el tipo de plan que guió el proceso de producción. Los primeros dos son fundantes, mientras que el plan es la función guía. Pienso que ésta es una buena aproximación al concepto de artefacto, pero necesita refinarse aún más. En primer lugar, la aproximación presupone que la materia bruta o prima usada en la producción de un artefacto es modalmente homogénea, pues de otra manera no tendría base hablar de el tipo de material natural. Sin embargo, de hecho, en uno y el mismo artefacto pueden entrar simultáneamente como partes o subtodos materiales que tienen funciones cualificadoras en distintas modalidades. No hay razón, por lo tanto, para no decir que un artefacto puede tener más de dos funciones fundantes al mismo tiempo. Llamemos funciones materiales a aquellas modalidades que cualifican los materiales a partir de los que está hecho el artefacto. La modalidad que cualifica el proceso de producción será llamado función técnica. La función guía es - como antes-el plan que guía la producción del artefacto. Al complejo consistente en la función o funciones fundantes de una hipóstasis (cuando tales existen), junto con su función guía, será llamado función cualificadora. Se infiere que, en las cosas naturales, la función guía y la función cualificadora coinciden.

Hemos caracterizado así los artefactos humanos usuales. Pero hay otros artefactos humanos que tienen peculiaridades especiales, pues están construidos precisamente a partir de seres humanos. Las estructuras sociales son estos artefactos, los cuales se caracterizan porque, en ellos, las funciones materiales están ausentes, pues están hechos de humanos y los humanos carecen de función cualificadora. Así, en general, por artefacto (humano) se entiende una hipóstasis que es producto de la actividad humana de acuerdo con un plan, que tiene al menos una función técnica y una guía. El artefacto es social si su función cualificadora incluye sólo una función técnica y una función guía. Es llamado no social o usual si también incluye funciones materiales. Cualquier actividad humana que produce artefactos será llamada un proceso de trabajo o de producción cultural.

A partir de este concepto de artefacto es posible elaborar toda una filosofía política y económica, pero ello rebasa las finalidades del presente trabajo. El lector interesado en estos temas está invitado a leer García de la Sienra (1994, 1994a), Clouser (1991) y, desde luego, la gran obra de Herman Dooyeweerd (1985). 


\section{El orden causal}

La metafísica aristotélica habla de cuatro causas del ente: la material, la formal, la eficiente y la final. Casi todas las escuelas contemporáneas de filosofía restringen el término "causa" a algo semejante a la causa eficiente. Tomo aquí el término "causa" en este sentido más contemporáneo, a saber, para designar el hecho evidente de que las cosas son creadas, producidas o generadas por otras cosas. Esto no es negar que puedan señalarse, en la causación, factores análogos a los que los aristotélicos llaman causas materiales, formales y finales, sino sólo tomar una decisión lingüística para apegarse al uso filosófico ordinario actual. Las cosas que crean, generan o producen son llamadas "causas" por esa razón, aunque en el uso actual del término se entiende por causa un evento que da lugar inmediatamente a un efecto. Por ejemplo, es más usual decir que la causa del mueble es la acción de producirlo, que decir que es el carpintero; en tales casos, a veces se dice que el carpintero es el causante del mueble. Como lo dice Sir David Ross al explicar a Aristóteles:

Podemos establecer la causa de un efecto $B$ o bien como $A$, el dueño de la facultad, o bien como " $A$ que ejercita la facultad". La causa de que una casa esté siendo construida es o bien "un constructor", o bien "un constructor que construye". ${ }^{10}$

Estos usos ordinarios sugieren una distinción entre el proceso mediante el que se crea, produce y genera algo, por un lado, y los agentes que intervienen en dicho proceso. Esta distinción se aprecia con toda claridad en el proceso de trabajo, donde típicamente intervienen - como señalaba Carlos Marx en El capital - fuerza, medios, y objetos de trabajo. ${ }^{11}$ Los objetos de trabajo fueron conceptualizados por Aristóteles como la causa material o materia (segunda) del ente ( $v . g r$. el bronce de una estatua); la fuerza de trabajo (el escultor que esculpe) correspondería a la causa eficiente; a grandes rasgos, la causa final corresponde a lo que nosotros llamamos "función guia" (la representación estética); la causa formal, finalmente, la cual según Marx existe "idealmente" en la mente del obrero al comienzo del proceso de trabajo, corresponde a grandes rasgos a ciertas propiedades físicas del ente (como la configuración de la estatua).

Desde luego, no todos los agentes de una transformación son humanos y por ende poseedores de una intencionalidad. Pero, de todos modos, el proceso de trabajo es un modelo a partir del cual se puede obtener por generalización la estructura de la causalidad. Notemos, en primer lugar, que se habla de causalidad siempre que estamos ante un proceso de transformación. El proceso de trabajo es un proceso de transformación especial.

10 Ross (1966), p. 72. La traducción es mía.

11 Véase El capital, volumen 1, capítulo V, y García de la Sienra (1980), p. 78. 
Podemos decir, por ende, que la causalidad es al menos parte de la estructura interna de un proceso de transformación. Pero un proceso de transformación es un ente individual, pues es algo que realmente tiene lugar en el tiempo. Luego, es enteramente natural pensar que la causalidad operante en el proceso está gobernada por la ley tipo del mismo, y que los factores del proceso son partes o subtodos del mismo.

Un proceso de transformación es un proceso en el que nuevos entes individuales son producidos, o en el que las propiedades potenciales de ciertos entes se activan. En todo proceso de transformación $P$ podemos distinguir (1) los materiales del proceso, los cuales existen previamente a $P$ y sobre los cuales actúan (2) las causas eficientes, que consisten en entes individuales con ciertas capacidades de operación y/o predisposiciones (propiedades) que los hacen reaccionar de cierto modo ante los materiales, lo que da lugar al (3) producto resultante. Las capacidades de operación de las causas reaccionan de ese modo peculiar ante los materiales en virtud de conexiones nómicas entre éstas y los materiales. Estas conexiones pueden ser normativas, como cuando un obrero determina operar de cierta manera sobre sus objetos de trabajo. Pero incluso en ese caso, en el que el fundamento de la conexión es la voluntad de una persona, la conexión asume forma de ley. Por eso, Marx podía decir que el objetivo del trabajador, del cual él mismo es consciente, "determina, como una ley, el modo y manera de su accionar y tiene que subordinar su voluntad al mismo". ${ }^{12}$ Estas conexiones nómicas están contenidas en la ley tipo del proceso $P$.

Nada impide, finalmente, que veamos el devenir entero del cosmos como un magno proceso de transformación. Religiones como el budismo ven este proceso como repetitivo, como un eterno retorno de las cosas a su principio. El materialismo lo ve como un proceso lineal sin principio ni fin. El judaísmo y el cristianismo lo ven también como un proceso lineal, pero con un principio y un fin. Ninguna de estas posiciones religiosas -0 cualquier otra- puede teoréticamente justificarse. Sin embargo, todo teorizar está guiado por alguna creencia religiosa $u$ otra, aunque sea a modo de una presuposición inconsciente. Desde una perspectiva cristiana, en el principio del proceso nos encontramos una hylé primigenia pero no concebida al modo de la materia prima aristotélica, sino consistente en entes individuales (¿partículas de hidrógeno?) dotados de propiedades y sujetos a leyes. El fin es concebido como un estado en el que el tiempo "ya no será más". Y, por ende, los procesos intermedios entre el principio y el fin son vistos como procesos parciales de transformación que forman algo así como un grafo que converge al estado final.

12 La cita original en la edición de Scarone reza: "determina, como una ley, el modo y manera de su accionar y al que tiene que subordinar su voluntad", pero prefiero el giro de la frase modificada. Cfr. Marx (1990), p. 216. 
Dentro de la visión judeocristiana, las leyes tipo del cosmos son las que rigen o norman la paulatina aparición de las formas bióticas, psíquicas y sociales. Ello sólo es posible si desde el principio está dado el marco nómico en su integridad y coherencia, y ésta es una de las razones por las que la filosofía de la idea cosmonómica rechaza toda forma de reductivismo.

BIBLIOGRAFIA

Beuchot, M., 1987, Metafísica. La ontología aristotélico-tomista de Francisco de Araújo, UNAM, México.

Bunge, M., 1967, Scientific Research I. The Search for System, Springer-Verlag, Berlín. Clouser, R.A., 1991, The Myth of Religious Neutrality, University of Notre Dame Press, Notre Dame.

Dooyeweerd, H., 1985, A New Critique of Theoretical Thought, 4 vols., Paideia Press, Jordan Station (Ontario).

García de la Sienra, A., 1980, "Elementos para una reconstrucción lógica de la teoría del valor de Marx", Crítica, no. XXXV, pp. 71-95.

_, 1994, "The Modal Laws of Economics", sometido a Philosophia Reformata, Amsterdam.

—, 1994a, "Toward a Cosmonomic Ontology of Economics", sometido a The Metaphysics of Economics, Helsinki.

Gracia, Jorge J. E., 1988, Individuality, State University of New York Press, Albany. Johansson, I., 1989, Ontological Investigations, Routledge, Londres.

Kalsbeek, L., 1975, Contours of a Christian Philosophy, Wedge, Toronto.

Krantz, D. H., R. D. Luce, P. Suppes y A. Tversky, 1971, Foundations of Measurement $I$, Academic Press, Nueva York.

Marx C., 1990, El capital, t. I, vol. 1, edición de P. Scarone, Siglo XXI, México.

Ross, D., 1966, Aristotle. Methuen, Londres. 